\title{
[Reseña de congreso] \\ Reseña del IV Congreso Internacional de Investigación Educativa en Chihuahua
}

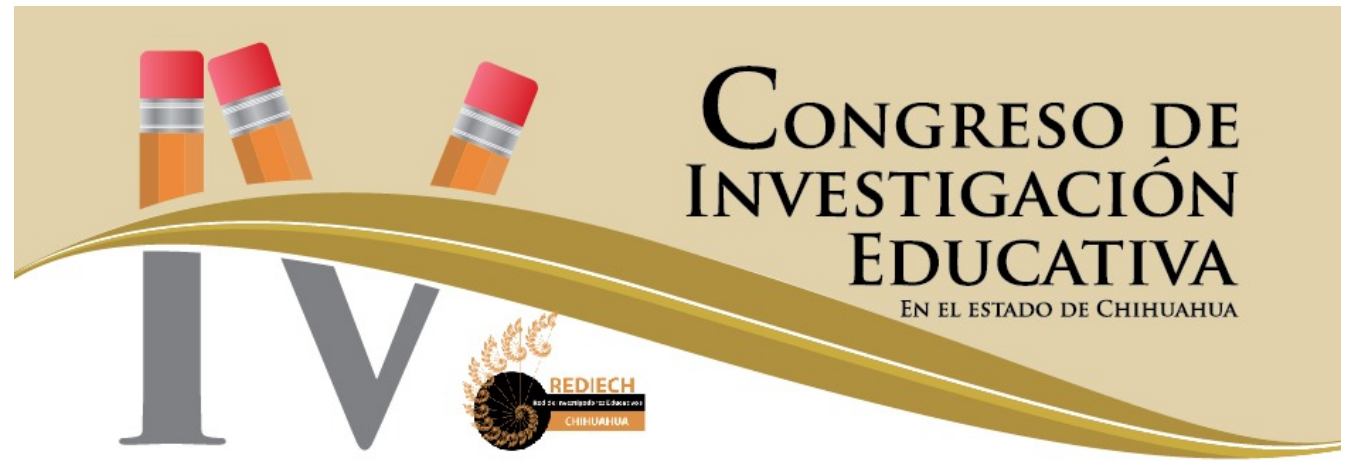

María de Lourdes González Peña ${ }^{1}$

Del 11 al 13 de octubre de 2018 se realizó en las instalaciones de la Facultad de Filosofía y Letras de la Universidad Autónoma de Chihuahua, el IV Congreso Internacional de Investigación Educativa, organizado por la Red de Investigadores Educativos Chihuahua (REDIECH). El evento se centró en los logros y desafíos de la investigación educativa como herramienta para la transformación social (Trujillo, 2018). En esta reseña parece oportuno hacer un balance de las principales actividades desarrolladas durante los tres días del evento.

La conferencia inaugural es digna de comentar porque se enfocó en ofrecer -tanto a investigadores como a asistentes- soluciones para lograr mayor impacto en las prácticas pedagógicas. La disertación tuvo por título "Enseñar o el oficio de aprender. Docentes para el siglo XXI", a cargo del distinguido investigador español, Dr. Miguel Ángel Santos Guerra, quien propuso 7 puntos para impactar en los aprendizajes de los estudiantes. En primer término, sugirió partir de la incertidumbre y centrarse menos en la

1 María de Lourdes González Peña. Investigadora independiente, Cuernavaca, México. Sus líneas de investigación son: diseño y validación de instrumentos de evaluación, proyectos formativos y desarrollo de competencias y análisis y validación de estrategias didácticas. Correo electrónico: lugmx77@gmail.com

ID: https://orcid.org/0000-0003-2431-4090 
certeza, enfatizando los riesgos de usar el lenguaje como medio de dominio y poder. En este sentido hizo alusión a la hermenéutica de Heidegger y Gadamer y a la Hermenéutica crítica de Habermas (Herrera, 2003; Santos, 2003 y 2014).

El segundo punto propuesto fue transitar de la simplicidad a la complejidad, señalando que las estrategias e instrumentos de evaluación pueden utilizarse como recursos para que los estudiantes revisen su desempeño y valoren el logro de las metas que se proponen (Morin, 2004; Santos, 2014).

El tercer punto se refirió a los beneficios de la colegialidad y las dificultades de actuar con individualismo, acentuando que parte de los compromisos que tiene el profesorado es mejorar las condiciones de vida de los estudiantes (Santos, 2015 y 2017). El cuarto punto lo enfocó en su propuesta para saltar de la neutralidad al compromiso, señalando que esto no obedece a una simple moda porque innovar o ajustar las acciones es un requisito indispensable para encontrar soluciones viables a los retos que se presentan en diferentes contextos (González, 2015; Santos, 2015).

En el quinto punto, Santos Guerra propuso pasar de la homogeneidad a la diversidad, dado que no todos los estudiantes poseen la misma información y por ello sugiere analizar los instrumentos de evaluación que se aplican para que la escuela no sea participe del fracaso de los jóvenes (Santos, 2014 y 2015). En el sexto punto señaló la importancia de pasar de la queja a la transformación, pues aunque puede haber motivos para el descontento por parte de los académicos (por relaciones verticales asfixiantes en el interior de la institución donde se presta el servicio, por tratos injustos o por las excesivas tareas administrativas con poco impacto en el desempeño de los estudiantes), aun así hay algunos docentes que realizan sus labores pensando que la decisión de mejorar está en sus propias manos (Santos, 2017).

Finalmente, en el séptimo punto el conferencista invitó a pasar de la frialdad a la emoción, pues son muchas las ventajas de procurar mejores condiciones de vida para uno mismo, aprendiendo a ser felices con lo que hacemos, pues de lo contrario la insatisfacción es una desgracia para quien la vive y es una crueldad para los estudiantes y demás personas que nos rodean (Santos y Beltrán, 2003; Santos, 2017).

Otra actividad digna de comentar del primer día de actividades es el panel conformado por los doctores Hugo Casanova Cardiel, Manuel Gil Antón y Jesús Adolfo Trujillo Holguín; destacados investigadores mexicanos expertos en educación. La exposición tuvo por título "La política educativa 
de cara al nuevo sexenio. ¿Qué reformar de la reforma constitucional de 2013?", donde abordaron el rumor de la posible desaparición del Instituto Nacional para la Evaluación de la Educación (INEE), dada la falta de claridad para justificar la utilidad e impacto de las evaluaciones aplicadas a los docentes y debido a la ausencia de una oferta de formación continua que sirva a los maestros para mejorar sus prácticas (Casanova, 2018). Se abordó también el tema de las pruebas estandarizadas y las estrategias aplicadas en algunos planteles educativos para obtener resultados más altos; además de la preocupación sobre el sustento teórico del Nuevo Modelo Educativo al señalar que no existe alguna referencia bibliográfica en dichos documentos que provenga de investigadores educativos afiliados al Consejo Mexicano de Investigación Educativa o de autores mexicanos de reconocida trayectoria investigativa (SEP, 2017a y 2017b). Los tres expertos enfatizaron que no solo hay que centrarse en el problema educativo, sino en las propuestas de mejora.

No menos importante y emotivo fue que durante el Congreso se realizó un homenaje al Dr. Eduardo Flores Kastanis. Los asistentes, colegas y quienes tuvieron la oportunidad de conocerlo rindieron un merecido tributo a quien dedicó su vida a la investigación educativa y que además gozó de un notable aprecio. Fue así que el día continúo entre ponencias, presentaciones de libros y otras actividades.

En el segundo día se ofreció una conferencia a cargo del investigador colombiano Sergio Tobón, titulada "La nueva reforma educativa y la importancia de los enfoques pedagógicos desde la investigación latinoamericana". La discusión se centró en proponer la estrategia de los proyectos con enfoque socioformativo como solución ante el problema de la evaluación en los aprendizajes de los estudiantes (Tobón, González, Nambo y Vázquez, 2015). También señaló que es necesario mejorar las taxonomías que se proponen para enunciar los aprendizajes esperados porque regularmente usan como máximo nivel de logro la explicación, aunque lo deseable sería evaluar la implementación y la mejora continua (SEP, 2017b).

Tobón señaló tres momentos que caracterizan a los proyectos: a) la planeación flexible, b) la ejecución adaptable y c) la autocrítica para la mejora. Particularmente subraya que los proyectos se componen de: a) sensibilización y motivación; b) resolución de problemas de contexto; c) formación del proyecto ético para mejorar las condiciones de vida, el cuidado del medio ambiente, la prevención del racismo, entre otros; d) la evaluación socioformativa, referida a una valoración continua mediante clases modelo, socializar ejemplos; y e) dar evidencia de mejoras hasta lograr los aprendizajes esperados (Tobón, Cardona, Vélez y López, 2015). 
Por su parte, el Dr. Hugo Casanova Cardiel -destacado investigador, catedrático de la Universidad Nacional Autónoma de México (UNAM) y especialista en políticas educativas- cerró el segundo día con la conferencia titulada "La educación y los retos del nuevo sexenio", donde compartió con el público reflexiones en torno a las demandas de infraestructura que prevalecen en las instituciones educativas, sobre la necesidad de pasar del discurso a la mejora de la realidad educativa y en relación a los cambios superficiales en la política educativa.

En el tema de la cobertura, Hugo Casanova resaltó las necesidades de los adolescentes que son madres o padres de familia y que se les dificulta la asistencia a las aulas regulares; por lo que precisan de mejores oportunidades de acceso y permanencia. En términos generales fue una conferencia que acaparó la atención de los participantes y muestra de ello fueron la cantidad de preguntas realizadas, donde el doctor Casanova contestó con mesura, de forma sencilla y -a la vez- tomando nota de los tópicos que ofrecían como tema de discusión.

El tercer y último día de Congreso culminó con el panel "La investigación basada en los procesos de productos artísticos" a cargo de los doctores Robert L. Ransom Cartey -director de la Facultad de Artes- Adán Sáenz Díaz e Iván Sparrow Ayub. Cada uno ejemplificó la forma en que se puede motivar a los estudiantes para articular la música, pintura, teatro y arte en las clases. Señalaron también que los principales problemas en el área son pasar del individualismo a la colaboración y traducir en números la evaluación realizada a la producción artística.

El Congreso cumplió con los objetivos planteados por la calidad y profesionalismo de sus ponentes, conferencistas y panelistas. Los temas abordados resultaron relevantes por su cercanía a la realidad del maestro y a la situación cotidiana de la escuela.

\section{Referencias}

Casanova, H. (2018). La educación y los retos de 2018: una visión académica. México: UNAM.

González, J. (2015). Modificación de neutralidad y crisis de las ciencias europeas: sobre la posibilidad de una vida de la razón. Investigaciones fenomenológicas, (5), 143-157.

Herrera, D. (2003). Fenomenología y hermenéutica. México: UPN.

Morin, E. (2004). La epistemología de la complejidad. España: Pedro Gómez.

Santos, M. (2003). Trampas en la educación: el discurso sobre la calidad. España: Libros Arco la muralla. 
Santos, M. (2014). La evaluación como aprendizaje. Cuando la flecha impacta en la diana. España: Narcea.

Santos, M. (2015). Las feromonas de la manzana. El valor educativo de la dirección escolar. España: Grao.

Santos, M. (2017). Evaluar con el corazón. De los ríos de las teorías al mar de la práctica. España: Homo Sapiens.

Santos, M. y Beltrán, R. (2003). Conocimiento, ética y esperanza. España: Universidad de Málaga.

SEP. (2011). Plan de Estudios 2011. Educación Básica. México: Secretaría de Educación Pública.

SEP. (2017a). Modelo educativo para la educación obligatoria. México: Secretaría de Educación Pública.

SEP. (2017b). Aprendizajes clave para la educación integral. México: Secretaría de Educación Pública.

Trujillo, J.A. (2018). Indicios de consolidación de la investigación educativa en Chihuahua. IE Revista de Investigación Educativa de la REDIECH, 9(17), pp. 4-8.

Tobón, S., Cardona, S., Vélez, J.B., y López, J. (2015). Proyectos formativos y desarrollo del talento humano para la sociedad del conocimiento. Acción pedagógica, 24(1), pp. 20-31.

Tobón, S., González, L., Nambo, J., y Vázquez, J. (2015). La socioformación: un estudio conceptual. Paradigma, 36(1), pp. 7-29. 
RECIE. Revista Electrónica Científica de Investigación Educativa Vol. 4, núm. 2, enero-diciembre 2019, pp. 1401-1405. 